\title{
CONCERNING THE DEVELOPMENT OF THE EVERSHED MOTION IN SUNSPOTS*
}

\author{
Martin D. Altschuler, Yoshinari Nakagawa, and Carl G. Lilliequist \\ (High Altitude Observatory, \\ National Center for Atmospheric Research, \\ Boulder, Colo., U.S.A.)
}

\begin{abstract}
A BSTRACT
The non-linear, partial differential equations of magnetohydrodynamics are iterated simultaneously by computer to determine the time development of a single sunspot. Axisymmetry and incompressibility are assumed. The initial conditions are (1) zero velocity everywhere, and (2) the magneticfield distribution of a ring current embedded in the photosphere. The initial magnetic field is then allowed to relax by magnetic diffusion and by the creation of a velocity field. It is shown that (1) Evershed motion outward from the sunspot will develop from reasonable initial parameters, and (2) the growth rate of the magnetic configuration depends on the strength of the initial magnetic field.
\end{abstract}

One of the most interesting problems concerned with the sunspot phenomenon is the origin of the Evershed motion. In an attempt to find some clues to this problem, we have examined the MHD equations in the non-linear, time-dependent form by numerical methods. The magnetic and velocity fields are strongly coupled with one another through the non-linear terms in the differential equations. Magnetic forces accelerate the fluid via the $\mathbf{J} \times \mathbf{B}$ term in the equation of motion and the fluid flow distorts the magnetic field via the $\nabla \times(\mathbf{v} \times \mathbf{B})$ term in the equation for magnetic field. We limit ourselves to an incompressible medium and to an axisymmetric sunspot. We neglect temperature gradients. Our primary concern is the acceleration of fluid by $\mathbf{J} \times \mathbf{B}$ forces and the distortion of the magnetic field by the fluid motions. Our initial conditions (i.e. at time $t=0$ ) are (1) zero velocity everywhere, and (2) the magneticfield distribution of a ring current embedded in the photosphere. The initial magnetic field is then allowed to dissipate by both magnetic diffusion and the creation of a velocity field.

Our abstraction from a real sunspot to an axisymmetric ring current in an incompressible fluid with no temperature gradients is somewhat severe. However, the advantage of a simple calculation is that we know exactly what physics has been included and what assumptions have been made. We can therefore determine in detail the interactions of a few physical parameters.

* Presented by M. D. Altschuler.

Kiepenheuer (ed.). Structure and Development of Solar Active Regions, 193-200. C I.A.U. 
In the absence of temperature gradients and density gradients, there are no pressure gradients to restrain the $\mathbf{J} \times \mathbf{B}$ forces, and a magnetostatic model of a sunspot cannot evolve.

The equations that we wish to solve are (Gaussian units)

$$
\begin{gathered}
\begin{array}{c}
\partial \mathbf{B} \\
\partial t
\end{array}=\nabla \times(\mathbf{v} \times \mathbf{B}-\eta \nabla \times \mathbf{B}) \\
\frac{\partial(\boldsymbol{\nabla} \times \mathbf{v})}{\partial t}=\nabla \times\left[\frac{1}{4 \pi \rho}(\boldsymbol{\nabla} \times \mathbf{B}) \times \mathbf{B}-(\boldsymbol{\nabla} \times \mathbf{v}) \times \mathbf{v}+v \nabla^{2} \mathbf{v}\right] \\
\boldsymbol{\nabla} \cdot \mathbf{v}=0
\end{gathered}
$$

where $\eta$ is the magnetic diffusivity, $v$ is the viscosity (assumed constant), and $\varrho$ is the density. A constant force of gravity and a scalar pressure may be included in Equation (2), but they have absolutely no effect on the time development of the magnetic and velocity fields in a constant density (homogeneous) atmosphere, and vanish when the curl operator is applied. By the same token, only the tension due to the curvature of the magnetic field affects the velocity.

To simplify the geometry (and decrease the number of equations), we assume the sunspot to be axisymmetric, the solar plasma to be incompressible, and the azimuthal fields to be zero. Equations (1) and (2) can then be written in cylindrical coordinates in the scalar form (Chandrasekhar, 1956)

$$
\begin{aligned}
& \frac{\partial P}{\partial t}=\eta \Delta_{5} P-\frac{1 \partial\left(r^{2} P, r^{2} U\right)}{r^{3}} \frac{\partial(z, r)}{\partial(z)}
\end{aligned}
$$

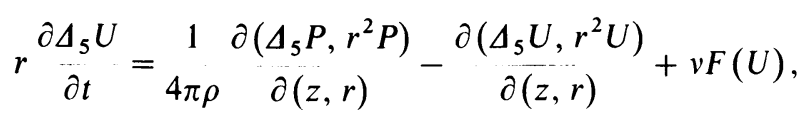

where

$$
\begin{aligned}
& F(U) \equiv r^{\partial^{2}\left(\Delta_{5} U\right)} \frac{1 \partial^{2}}{\partial z^{2}}+\begin{array}{l}
r \partial r^{2} \\
\left.r{ }^{2} \Delta_{5} U\right)-{ }_{r^{2} \partial r}^{1} \partial
\end{array}\left(r^{2} \Delta_{5} U\right) \\
& \frac{\partial(f, g)}{\partial(z, r)} \equiv \frac{\partial f}{\partial z} \frac{\partial g}{\partial r}-\frac{\partial f}{\partial r} \partial g
\end{aligned}
$$

and

$$
\Delta_{5} \equiv \frac{\partial^{2}}{\partial r^{2}}+\frac{3}{r} \partial r+\frac{\partial^{2}}{\partial z^{2}},
$$

where $z$ corresponds to the sunspot axis. The magnetic and velocity vectors are related to the scalars $P$ and $U$ by the relations

$$
\mathbf{B}=-r\left(\begin{array}{l}
\partial P \\
\partial z
\end{array}\right) \hat{\mathbf{r}}+\left[\begin{array}{lll}
1 & \partial \\
r & \partial r & \left(r^{2} P\right)
\end{array}\right] \hat{\mathbf{z}}
$$




$$
\mathbf{v}=-r\left(\begin{array}{l}
\partial U \\
\partial z
\end{array}\right) \hat{\mathbf{r}}+\left[\begin{array}{ll}
1 & \partial \\
r & \partial r
\end{array}\left(r^{2} U\right)\right] \hat{\mathbf{z}}
$$

Our task is to iterate Equations (4) and (5) simultaneously by numerical methods.

Our initial magnetic field is essentially that of a ring current with a radius of 1000 $\mathrm{km}$, a current of $2 \times 10^{11}$ ampères, and a dipole moment of $6.3 \times 10^{26}$ gauss $\mathrm{cm}^{3}$ $\left(=6.3 \times 10^{23} \mathrm{amp} \mathrm{m}^{2}\right)$. We have modified the field in the region immediately surrounding the ring to avoid the singularity.' Figure 1 shows our assumed initial magnetic field. The highest field strength (at the point marked $H$ ) is 1800 gauss. The initial velocity field is chosen to be zero at every point. The density is taken to be $\varrho=10^{-7}$ $\mathrm{gm} / \mathrm{cm}^{3}$, the viscosity to be $v=10^{12} \mathrm{~cm}^{2} / \mathrm{sec}$, and the magnetic diffusivity to be $\eta=10^{10} \mathrm{~cm}^{2} / \mathrm{sec}$. The time scale for the diffusion of magnetic field across $1000 \mathrm{~km}$ is $L^{2} / \eta=10^{6} \mathrm{sec}$. Since we will be limited to only about 1 hour of development, magnetic diffusion does not affect the results of the calculation.

Figures $2 \mathrm{a}$ and $2 \mathrm{~b}$ show the magnetic and velocity fields after $10 \mathrm{sec}$ of development; Figures $3 \mathrm{a}$ and $3 \mathrm{~b}$ show the fields after $100 \mathrm{sec}$; Figures $4 \mathrm{a}$ and $4 \mathrm{~b}$ show the fields after

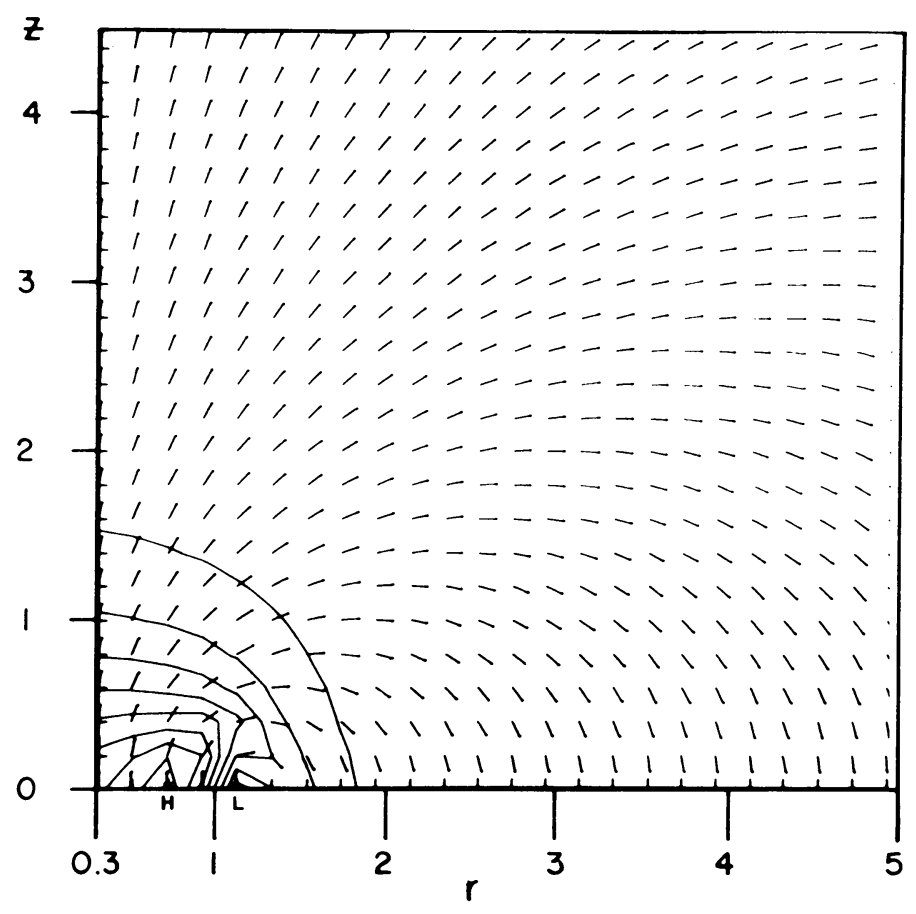

FIG. 1. The initial magnetic field. There is symmetry around the $z$-axis $(r=0)$ and with respect to the $z=0$ plane. The $r$-and $z$-axes are calibrated in units of $10^{3} \mathrm{~km}$. The $H$ (high) locates the maximum $B$ of 1780 gauss; the L (low) locates the minimum B of 70 gauss. The contours extend to 1600 gauss in steps of 200 gauss. 


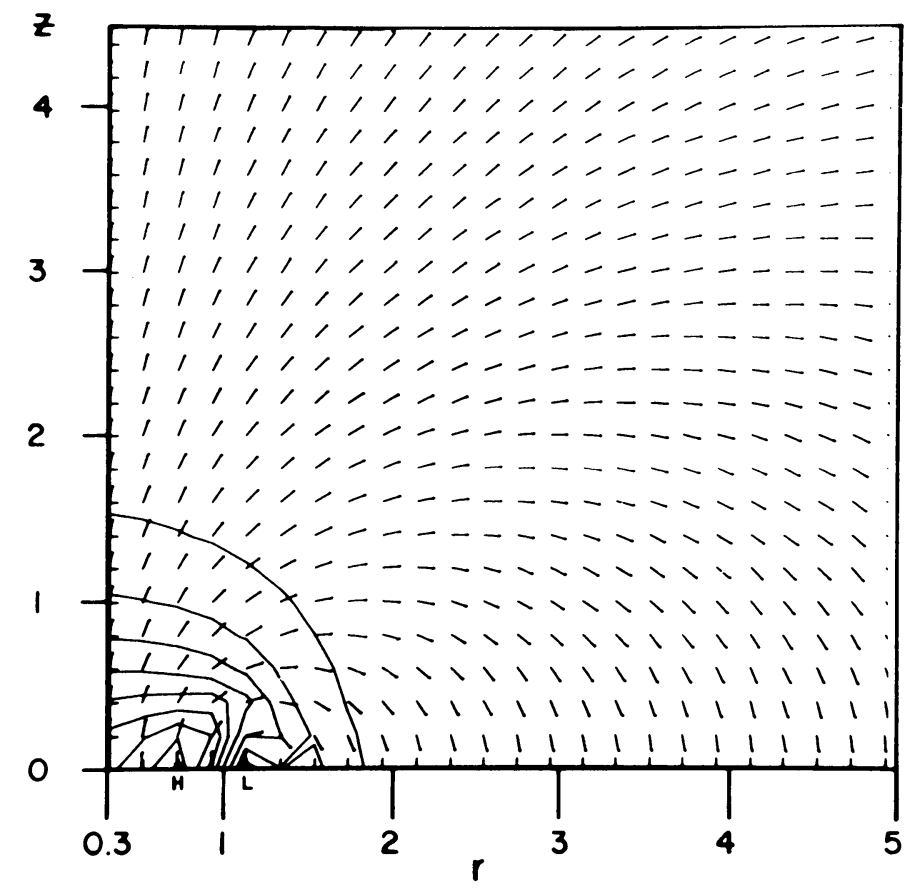

FIG. 2a. The magnetic field after 10 sec of development. Same scaling as in Figure 1. At $H$ the value of $B$ is 1750 gauss and at $L$ the value is 55 gauss.

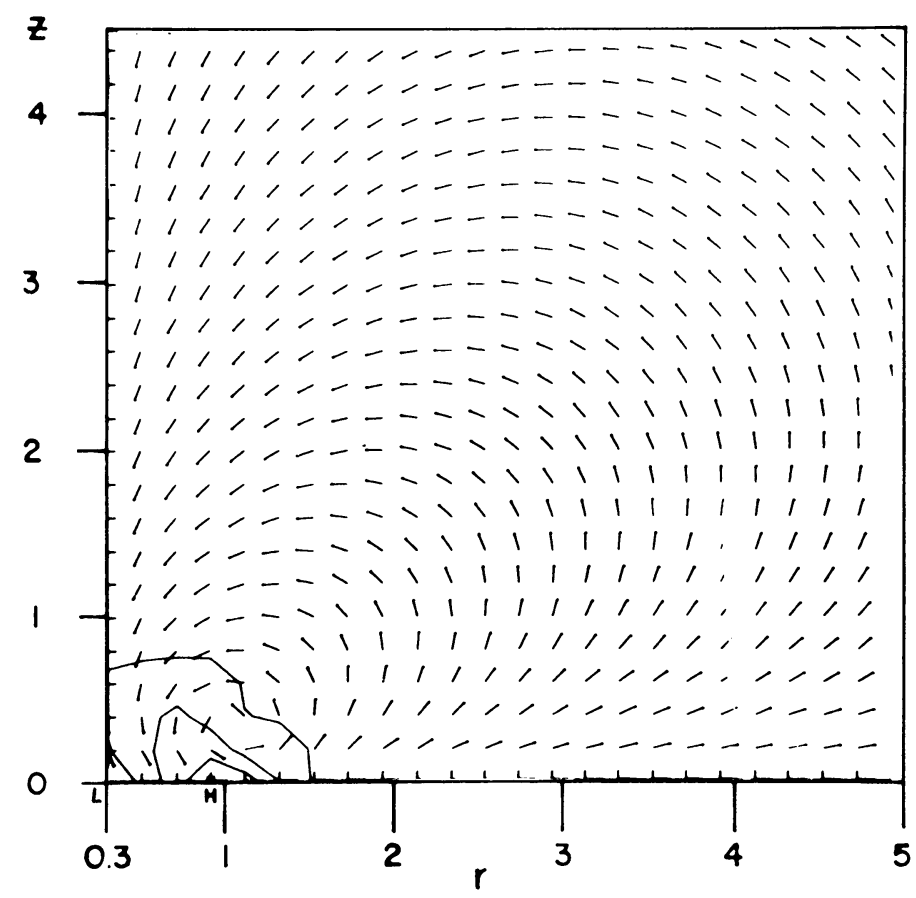

FIG. 2b. The velocity field after $10 \mathrm{sec}$ of development. The contours are in steps of $500 \mathrm{~m} / \mathrm{sec}$. At $H$ the maximum velocity is $1970 \mathrm{~m} / \mathrm{sec}$; at L the minimum velocity is $250 \mathrm{~m} / \mathrm{sec}$. 


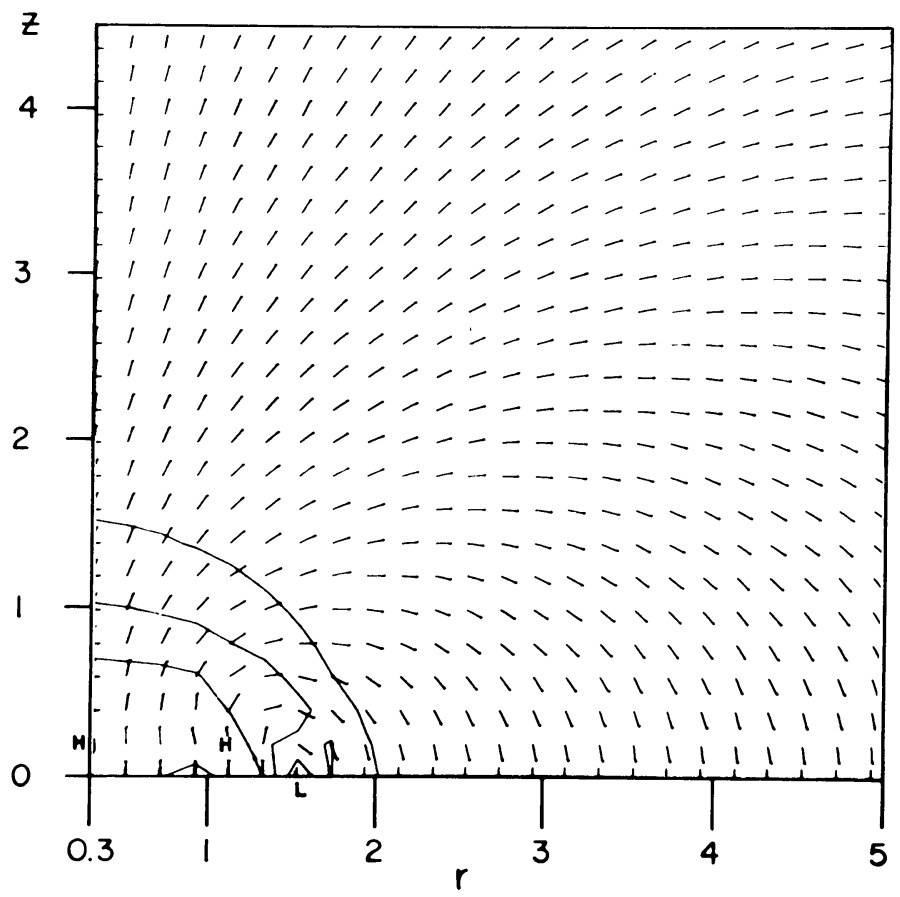

Fig. 3a. The magnetic field after 100 sec of development. Same scaling as in Figure 1. At $H(r=0 \cdot 3)$, $B$ equals 760 gauss; at $H(r=1 \cdot 1), B$ equals 820 gauss; at $L(z=0), B$ equals 20 gauss.

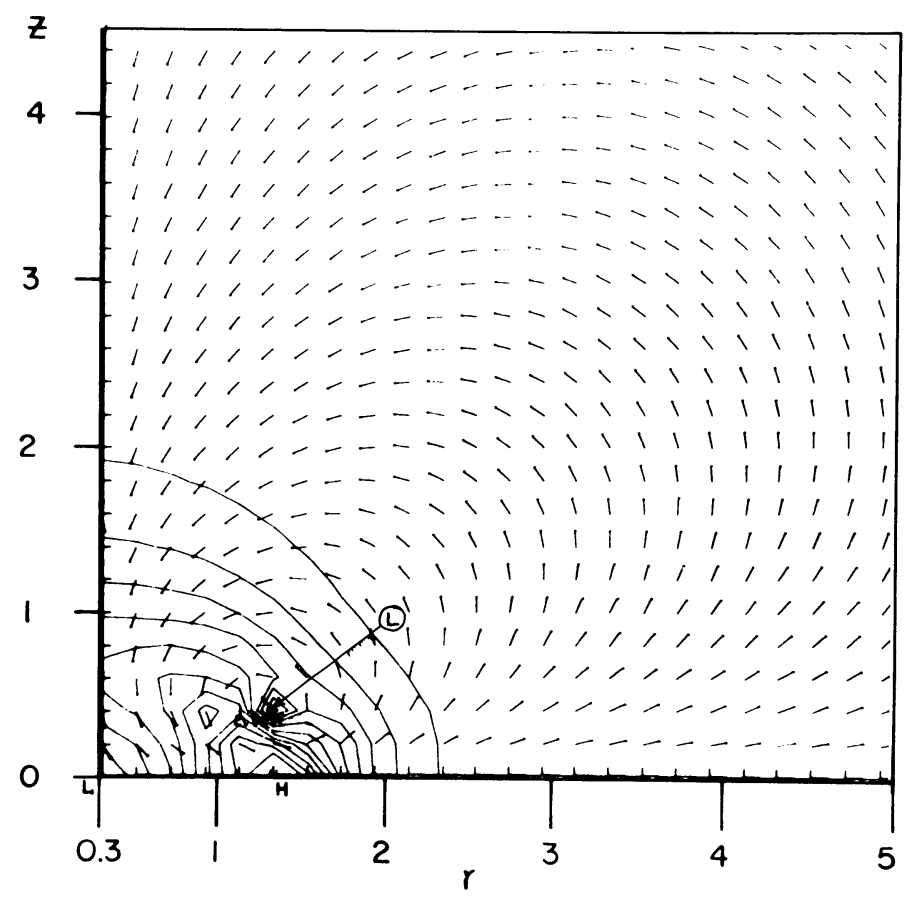

Fig. 3b. The velocity field after $100 \mathrm{sec}$ of development. Same scaling as in Figure 2b. At H, vequals $7130 \mathrm{~m} / \mathrm{sec}$; at $L(r=0 \cdot 3)$ i equals $120 \mathrm{~m} / \mathrm{sec}$; at $L(r .1 \cdot 3), v=210 \mathrm{~m} / \mathrm{sec}$. 


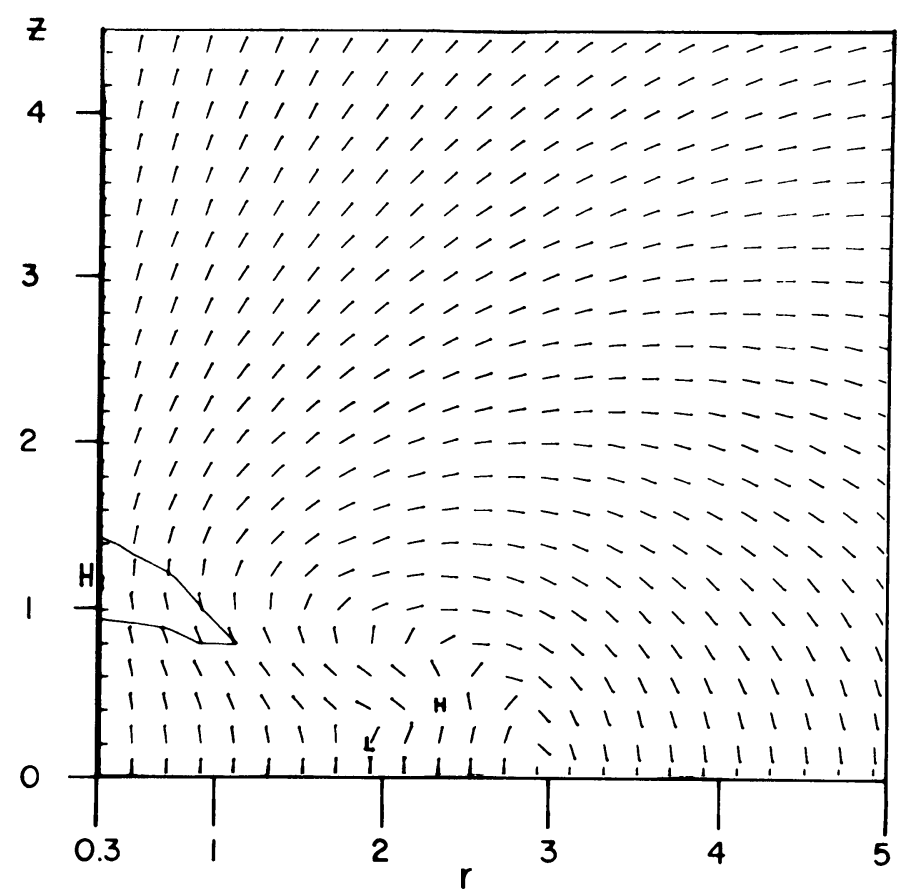

Fig. 4a. The magnetic field after 1000 sec of development. Same scaling as in Figure I. At $H(r=0 \cdot 3), B$ equals 220 gauss; at $H(r=2 \cdot 3), B$ equals 190 gauss; at $L, B$ equals 40 gauss.

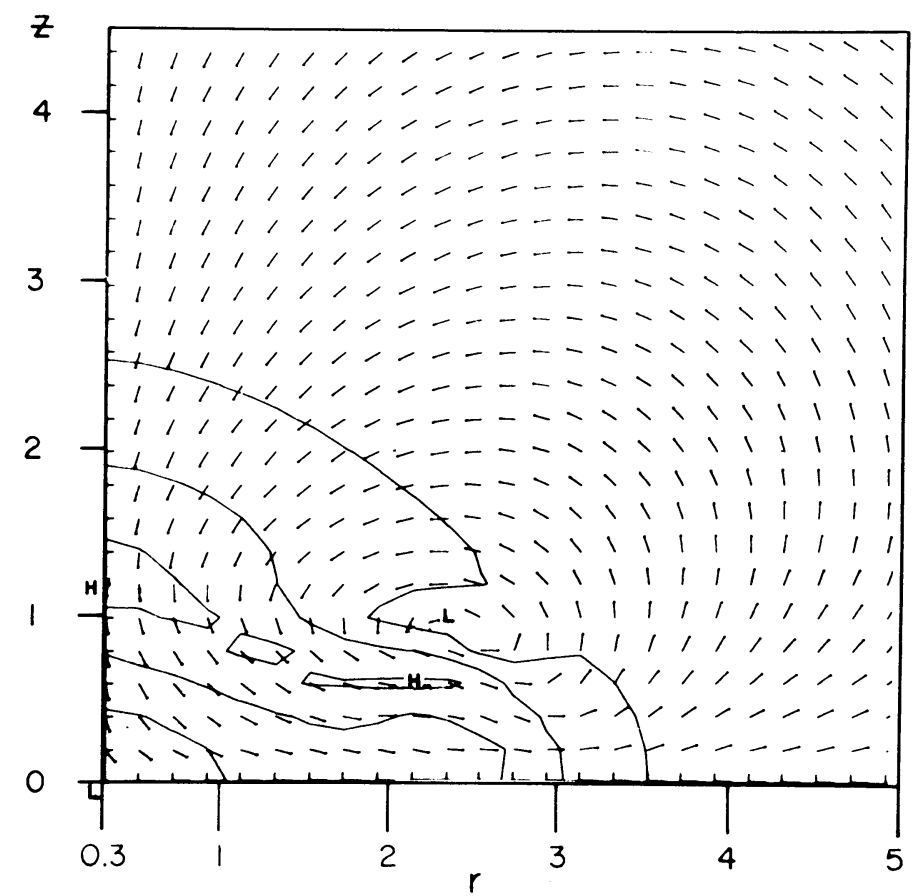

FIG. 4b. The velocity field after $1000 \mathrm{sec}$ of development. Same scaling as in Figure $2 \mathrm{~b}$. At

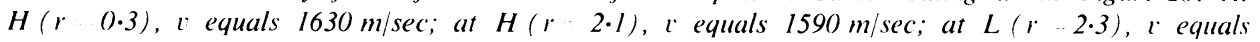
$210 \mathrm{~m} / \mathrm{sec}$. 
$1000 \mathrm{sec}$. Although we have carried the computation to $10^{4} \mathrm{sec}$, these early developments (in logarithmic time steps) show the actual development of the velocity pattern.

A careful examination of these diagrams indicates that the magnetic-field configuration is frozen to the moving fluid, and that the fluid velocity is approaching the Alfven velocity in the ring-current region. The magnetic field, although perpendicular to the velocity field at small $|z|$, is swept along by the flow. Near the sunspot axis, the magnetic and velocity fields are parallel.

The flow is radially outward from the sunspot axis for $|z|<1500 \mathrm{~km}$, and radially inward for $|z|>1500 \mathrm{~km}$. This result is independent of the magnetic polarity of the sunspot, and is strongly suggestive of the Evershed motion. The flow pattern resembles a vortex ring. As the magnetic configuration expands, the vortex ring expands in step.

This calculation, which applies to a large magnetic field and negligible magnetic diffusion (i.e., to Lundquist number $L u=v_{\mathrm{A}} L / \eta \gg 1$ ), shows that a strong (non-linear) interaction between the magnetic and velocity fields achieves an equipartition between the kinetic and magnetic-energy densities. The time scale for the growth of the magnetic configuration is $L / v_{\mathrm{A}}$, where $L$ is the scale length for magnetic variations $(\approx 1000 \mathrm{~km})$ and $v_{\mathrm{A}}$ is the Alfvén velocity.

A similar calculation for weak magnetic fields and large magnetic diffusion $(L u=$ $\left.v_{\mathrm{A}} L / \eta \ll 1\right)$ indicates that the drift of the magnetic field corresponds to a time scale $L^{2} / \eta$. Nevertheless, a small-magnitude Evershed flow pattern still results.

For different initial Lundquist numbers and field strengths, the magnetic field can develop toward dissimilar configurations.

Our calculations show that the existence of magnetic forces is a sufficient condition for the development of Evershed motion. Further work is needed to determine whether magnetic forces are also a necessary condition.

We conclude:

(1) The Evershed motion is probably the result of $\mathbf{J} \times \mathbf{B}$ forces which are radial to the axis of the sunspot in the photospheric region.

(2) The flow is perpendicular to the direction of magnetic field near the edge of the sunspot (where the current $\nabla \times \mathbf{B}$ is concentrated), and parallel to the magnetic field near the sunspot axis.

(3) The Evershed flow may be the most important factor in the decay of sunspots.

We have refrained from a detailed comparison of our results with observational data, because our assumption of incompressibility and the consequent omission of thermal pressure gradients are severe. Further development of our numerical model will eventually justify a thorough comparison of theory and observation.

\section{Reference}




\section{DISCUSSION}

Jäger: In your calculations what did you assume for the magnetic-energy density compared with the energy density of matter?

Altschuler: The initial condition is a medium of constant density which is nowhere in motion. As the magnetic-field configuration relaxes, magnetic energy is converted into kinetic energy by the $\mathbf{J} \times \mathbf{B}$ forces.

F. Meyer: I would like to add a remark of caution, if I may, with respect to the direct comparison of your computations with real sunspots. In your model you basically compute the development of a ring current in an incompressible medium. At the outset this current is in unequilibrium with the surroundings and then expands to achieve a lower energy configuration. This all occurs in a few hours, comparable to the traveling times of Alfvén waves. The much longer life-times of real sunspots indicate that they are basically in equilibrium with their surroundings. The forces leading to motions and changes here seem to be of convective type as evidence by the granulation, supergranulation and the penumbral convection rolls of Danielson. Thus it seems important to include the energy transport in the treatment. That this is much more complicated I think we all know.

Altschuler: Your points are well taken. We agree completely.

Sturrock: Your theory assumes that the Evershed effect is a transient phenomenon peculiar to the decay phase of sunspots. I believe that observations do not support this assumption. If the Evershed effect can occur in a sunspot which is virtually in a steady state, one must look for a power supply, not an energy supply. I suggest that the 'missing power' mentioned by Dr. Danielson, associated with the reduced radiation from sunspots, may in fact be the power which drives the Evershed effect.

Altschuler: We did this calculation in order to understand as much as possible about the behavior of a few physical parameters, rather than to understand only a little about the behavior of a great many parameters. If we could understand in detail how the missing power from sunspots is converted into Evershed motion, we would have much of the problem solved. 